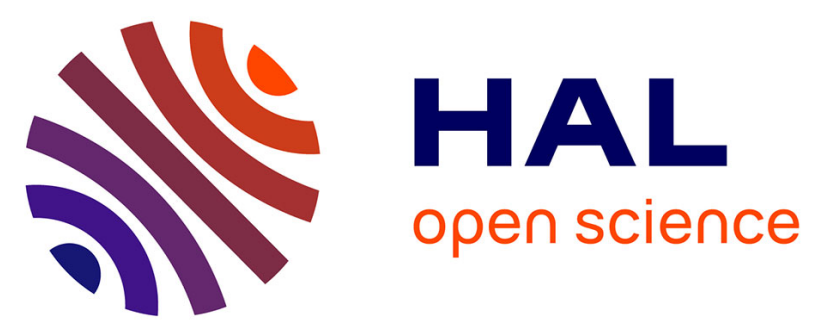

\title{
Simulation in Contexts Involving an Interactive Table and Tangible Objects
}

\author{
Sébastien Kubicki, Yoann Lebrun, Sophie Lepreux, Emmanuel Adam, \\ Christophe Kolski, René Mandiau
}

\section{- To cite this version:}

Sébastien Kubicki, Yoann Lebrun, Sophie Lepreux, Emmanuel Adam, Christophe Kolski, et al.. Simulation in Contexts Involving an Interactive Table and Tangible Objects. Simulation Modelling Practice and Theory, 2013, 31, pp.116-131. 10.1016/j.simpat.2012.10.012 . hal-00864161

\section{HAL Id: hal-00864161 https://hal.science/hal-00864161}

Submitted on 20 Sep 2013

HAL is a multi-disciplinary open access archive for the deposit and dissemination of scientific research documents, whether they are published or not. The documents may come from teaching and research institutions in France or abroad, or from public or private research centers.
L'archive ouverte pluridisciplinaire HAL, est destinée au dépôt et à la diffusion de documents scientifiques de niveau recherche, publiés ou non, émanant des établissements d'enseignement et de recherche français ou étrangers, des laboratoires publics ou privés. 
1

2

\title{
Simulation in Contexts Involving an Interactive Table and Tangible Objects
}

\author{
Sebastien Kubickia, ${ }^{\mathrm{a}}$, Yoann Lebrun ${ }^{\mathrm{a}}$, Sophie Lepreux ${ }^{\mathrm{a}}$, Emmanuel Adam ${ }^{\mathrm{a}}$, Christophe Kolski ${ }^{\mathrm{a}}$, René Mandiau ${ }^{\mathrm{a}}$ \\ ${ }^{a}$ Univ Lille Nord de France, F-59000 Lille, France, \\ UVHC, LAMIH, F-59313 Valenciennes, France, \\ CNRS, UMR 8201, F-59313 Valenciennes, France \\ ${ }^{b}$ Lab-STICC UMR 6285/ UEB / ENIB, \\ European Center for Virtual Reality, \\ Technopôle Brest-Iroise F-29280 Plouzané, France
}

\begin{abstract}
By using an interactive table, it is possible to interact with several people (decision-makers) in a simultaneous and collaborative way, around the table, during a simulation session. Thanks to the RFID technology with which the table is fitted, it is possible to give tangible objects a unique identity to include and to consider them in the simulation. The paper describes a context model, which takes into consideration the specificities related to interactive tables. The TangiSense interactive table is presented; it is connected to a Multi-Agent System making it possible to give the table a certain level of adaptation: each tangible object can be associated to an agent which can bring roles to the object (i.e., the roles are the equivalent of a set of behaviors). The Multi-Agent System proposed in this paper is modelled according to an architecture adapted to the exploitation of tangible and virtual objects during simulation on an interactive table. A case study is presented; it concerns a simulation of road traffic management. The illustrations give an outline of the potentialities of the simulation system as regards the context-awareness aspect, following both the actions of the decision-makers implied in simulation, and the agents composing the road traffic simulation.
\end{abstract}

Keywords: Simulation, interactive table, tangible objects, RFID, Multi-Agent System.

\section{Introduction}

\subsection{The problem: a need for more intuitive cooperative design tools}

For thirty years, many simulators have been proposed in a set of application areas [4]. Most simulation software are based on a standard architecture and can be used by a user interacting according to interaction principles, through the usual triplet <screen, keyboard, mouse $>[60,28]$. However, for implementing cooperative design approaches, related to dynamic situations, complex, multifaceted, it is necessary to provide interactive simulation tools that are more intuitive than the current tools, allowing different actors (e.g., decision-makers) to think together and react to changes in context. This paper explores interactive tables ${ }^{1}$, associated with tangible objects. Our case study concerns road traffic management.

\subsection{Interactive tables and tangible objects for a new simulation support}

With evolving technology, the objects in our environment will be increasingly equipped with computing capacities and memory, especially in the data processing domain in which the evolution has been significant. Little by little, data processing has been introduced into everyday life and has become known as ambient data processing [54]. By highlighting inter-object communication and making it possible for objects to perceive their environment; objects must have the "intelligence" (in the sense of artificial intelligence) to meet various objectives.

This technological progress lets us to imagine new simulation systems in which any type of interaction would be possible: the user becomes the primary interactor with the system, but the other people and objects that surround $\mathrm{him} / \mathrm{her}$ can also intervene in the simulation system.

\footnotetext{
${ }^{1}$ The interactive tables are also called tabletops or interactive tabletops in the literature.
} 
We propose to use pervasive technologies in order to increase interaction and intelligence in simulation systems, with the help of an interactive table. Interactive tables differ from the computer because they make collaborative or competitive group work possible. New interactions are thus possible and feasible. Nowadays, there are few applications and platforms, which allow simultaneous collaborations between users (e.g., multi-pointing or real-time document sharing). For this reason, current research aims to explore the possibilities of this new technology.

\subsection{Case study: road traffic management}

Traffic trials can last for several days, or even several weeks, in order to evaluate the infrastructures about the impact of new roads, motorway entries/exits, new roundabouts, and road signs, for example. In addition, researchers conduct studies into the psychological aspects. Human beings are included in the traffic loop, thus it is necessary to assess their behaviour in specific contexts.

Road traffic models can be distinguished by their design method: centralized and distributed. In centralized methods, mathematical models appeared in the 1950s. Such a model allows a highway traffic situation to be modeled using car-following laws, for instance. These laws are, in fact, differential equations that are obtained empirically through regression using data collected at road sections currently operating [34, 2]. Even now, most of the microscopic simulations use the car-following laws to model in-lane driving, while the specific case of intersections is managed using centralized scheduling techniques. In these simulations, each vehicle approaching the intersection is placed in a virtual queue, one for each branch of the intersection. For instance, Vissim [51] has a "yellow box" parameter, which allows users to define a minimal speed that the vehicles inside the intersection must respect so that other vehicles can enter the intersection. These traffic simulation tools suggest that a centralized scheduler makes decisions for each vehicle, which enters the intersection only when their trajectories are not in conflict. The simulated behaviors of individual drivers produced by schedulers are not always realistic, and thus many traffic phenomena can not be simulated (e.g., the presence of traffic signals violations and congestion inside the intersection).

Since the beginning of the 1980s, distributed methods, called also behavioral approaches, have highlighted a different view. These methods consider traffic as emerging phenomena, which result from actions and interactions of the various traffic system actors (e.g., drivers, pedestrians, road operators). Different models have been developed for building this traffic: cellular automata models [45], robotic-inspired models [43], and multi-agent models [8, 20].

For several years now, our team has focused primarily on these traffic models with multi-agent systems [24, 55]. We suppose that it is possible to propose new types of agent-based simulations on the interactive table described in this paper.

\subsection{The paper's contents}

In this paper, we introduce a new type of interactive table based on Radio Frequency IDentification (RFID) technology (Figure 1). This table allows the users to handle tangible objects equipped with RFID tags, enabling them to record information. The users can thus interact and work on new applications using tangible objects (e.g., design or production tasks, collaborative decision-making). This high-level technological platform (i.e., the interactive table) is a support for our research. Such platforms lead us to envisage new ways of approaching simulation, combining both virtual and tangible objects [32]. To be efficient, they need models that take into account the context of use. This adaptation to context can be modeled through a Multi-Agent System (MAS). Multi-agent systems are appropriate for this type of rich simulation environment because they are based, in particular, on models of distributed representation and reasoning [14]. Their deployment in an Ambient Intelligence situation [26] would adapt the intelligence of so-called everyday objects to both the users and the context of use.

In the next section, we present a state of the art about context-awareness and simulation. In section 3, we present the TangiSense interactive table and its software architecture. In section 4, we propose a multi-agent system (MAS) to manage the smart objects and context-awareness. This MAS allows remote operations of intelligent objects using a software representation. In section 5, we examine a case study based on a road traffic management simulation. Section 6 discusses our proposal. Section 7 gives our conclusions and our prospects for future research. 


\section{Previous research}

\subsection{Context-Awareness}

The context concept has been used in interactive application design since 1994. In 1994, Schilit et al. [46] introduced the concept, context-awareness, and associated it to a mobile system (ParcTab), in which user location ("Where are you?"), the identity of the people ("Who you are with?"), as well as the proximity of the resources ("What resources are nearby?") allow the context to be studied. They described how the applications can react to changes in the physical environment or the user. Some applications allow the users to participate and the users and devices to interact; however, these applications react in particular according to the context.

Ward et al. [53] interpreted the context using the user localization and the state of the environment. They also considered the localization of objects. Pascoe [41] defined the context-awareness concept, defining the context according to four generic contextual capacities: perception, adaptation, resource discovery, and contextual augmentation.

Dey et al. [17] added a detail to these concepts. They defined the context as a set of information elements making it possible to characterize the situation of an entity, which is a person, a place or an object that can intervene in the interaction between the user and the application. Thevenin \& Coutaz [48] defined the interaction context concept ${ }^{2}$, which is connected to the definition proposed by Dey et al. The environment becomes an entity triplet $<$ Object, Person, Event $>$ associated to the current task.

Calvary et al. [11] introduced the plasticity concept and the user interface adaptation. This adaptation is modelled as two complementary properties: adaptability and adaptivity [10,47]. Adaptability is the capacity of the system to allow users to adapt their systems starting from preset parameters; adaptivity is the capacity of the system to automatically improve the adaptation without user action.

In 2004, the context became context of use. Calvary et al. [12] put forward the adaptation of the Human-Machine Interfaces to their context of use, seen as a triplet: <User, Platform, Environment $>$. The user represents the public involved, the platform corresponds to the material and software structure underlying the interaction, and the environment refers to the physical environment supporting the interaction.

Rey et al. [44] proposed a context network, called the contextor. They also proposed a component model that allows the capture, transformation and diffusion of contextual information.

More recently, Dey et al. $[16,18]$ used similar concepts as those described by the previous authors (e.g., localization, state of the people in close proximity, time) to propose interactive prototyping of context-aware applications.

\footnotetext{
${ }^{2}$ Please note the term change.
} 
Pascoe et al. [42] added the concept of social context, which included the people's biometric signs, history, emotions, status and mood.

To summarize, the definitions in the state of the art refer to the localization and the physical environment, to the user and generally to a specific platform in order to define the context. However, little by little, the cited authors made some modifications that make it possible to specify and adapt precisely the applications to the context with some indications, such as entities, time or state. Nonetheless, current research (after 2007) on context-awareness does not modify the definitions suggested previously.

We propose to model the context criteria with a class diagram (Figure 2) [32]. The criteria most often mentioned in the references cited in this state of the art are arranged in classes. This classification uses the triplet $<$ User, Platform, Environment $>$ proposed by Calvary et al. [12]. The users can be characterized by their competence, ability, emotions and cultural information; their preferences can also be included. The characteristics of the platform are taken into account for the adaptation. The platform proposes a display surface for interaction (i.e., screen), which can be tactile or not. Finally, the environment includes the localization, the type of environment (i.e., social, professional or private), the resources available in the vicinity, the information on the external environment (e.g., local characteristics), and the possibility of using communication technologies. This model is sufficiently general to be adapted according to specific needs. It can be easily extended, depending on the development and use. It has been extended for using interactive tables. The related extensions are outlined on Figure 2. They are detailed in Section 4.1.

In this paper, we will focus on the concept of context-awareness, using an interactive table that makes it possible to act on virtual and/or tangible objects.

\subsection{Road traffic simulation based on multi-agent approaches}

The multi-agent model which agents evolve in traffic situations, as well as interactions between these agents. The main advantage of multi-agent models is based on the environment's dynamic modifications in a response time that is close to real time: preferences and characteristics of autonomous vehicles, appearance of vehicles (e.g., buses, motorbikes, cars), pedestrians and the road signs (e.g., stop signs, give way signs, speed-limit signs). The agents perceive information that is geographically limited and incomplete. The traffic situation is, by nature, an open system (i.e., the number of autonomous agents can vary during the simulation) in which the various entities do not cooperate with each other, each having their own objectives. The situation is defined by multiple interactions between entities in their environment, which makes it possible to reproduce more realistic behaviors of human drivers. In fact, simulation conditions can be dynamically modified: the degree of visibility from the weather, the driving preferences of the human driver, the characteristics of the autonomous agent (e.g., cars, lorries, buses, pedestrians) and road equipment (e.g., traffic signals, traffic signs).

Two research approaches are starting to be differentiated in terms of road traffic simulations using $M A S$, made by Meir and Rosenschein [37]. The first approach tries to offer organizational models to improve global problems, such as logistics and/or services [15,59], and the second approach offers solutions for "local" traffic congestion problems. Congestion is a deteriorated state because all agents make "optimum" local decisions a priori, far from the global optimum.

Several studies have tried to answer the problems of congestion. From these different studies, two multi-agent categories can be cited to model the traffic in critical situations. A first category deals with coordination models/protocols (e.g., simulation methods) or the equilibrium research [5,37]. These ideas are essentially based on the way to optimize global traffic. A minimal infrastructure is thus envisaged to regulate the agents/vehicles in an intersection or in an intersection network. A reservation mechanism $[22,50]$ provides a coordination protocol for managing the space in an intersection. Another method described by Trannois et al. [49] uses a Blackboard mechanism for scheduling the traffic in the intersection, and yet another method describes a mechanism to control traffic lights by minimizing conflicts between the different agents [25].

A second category describes "profiles" for the different agents, and then analyzes the impact of these profiles on the global traffic. These methods make it possible to obtain global information on the simulation (e.g., statistics data concerning the average speed of vehicles, the number of accidents) to compare with real observed data. For example, Ehlert and Rothkrantz [23] define agent profiles with a specific behaviour (i.e., prudent or aggressive behaviours, fast or slow driving). The agents update the different information from the environment, and they adapt their behaviour from a set of predefined rules. In similar approaches, the autonomous agents make decisions according to predefined 


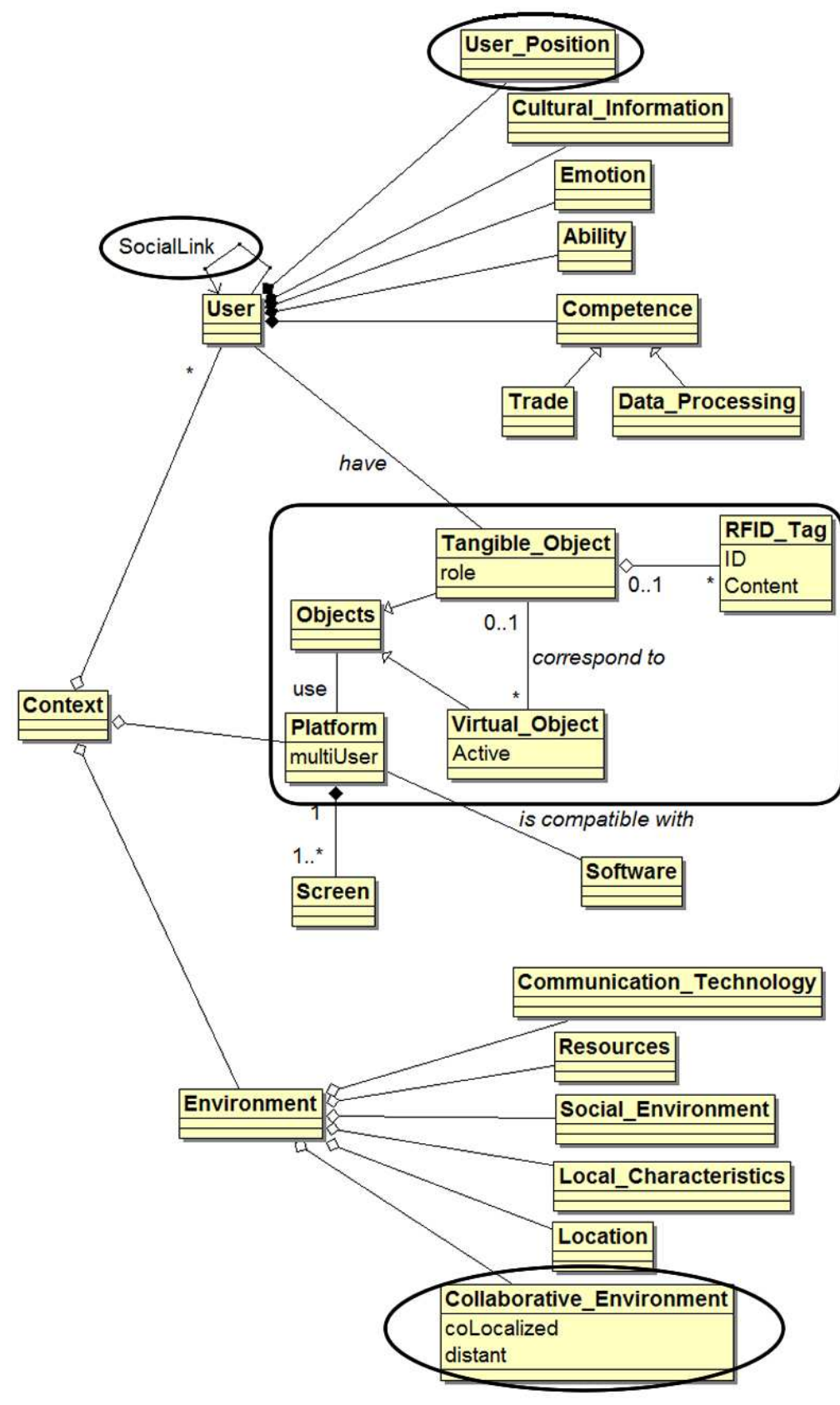

Figure 2: Modelling of the interaction context, with the new criteria concerning interactive tables (outlined with a bold-faced line)

behaviours (normal, prudent or aggressive) and different parameters (e.g., inter-vehicular distance or accelerationbreaking characteristics) [40], or make speed-acceleration decisions and set maximum speed [58]. The agents may also have non-normative behaviours (i.e., not respecting the highway code, not breaking at a stop sign) [20].

These studies are very widespread, with different goals (e.g., the optimization of global traffic or the understanding of human driving). We would like to describe a new simulation tool that is based on an interactive table. The simulation may be dynamically modified by the different users: changing the road infrastructure or altering the local behaviour of specific agents. 
Table 1: Five interactive tables using different capture technologies

\begin{tabular}{|c|c|c|c|c|c|c|c|}
\hline Name & $\begin{array}{c}\text { Capture } \\
\text { technology }\end{array}$ & Tactile & $\begin{array}{c}\text { User } \\
\text { distinction }\end{array}$ & $\begin{array}{c}\text { Object } \\
\text { detection }\end{array}$ & $\begin{array}{c}\text { Object } \\
\text { overlay }\end{array}$ & $\begin{array}{c}\text { Artificial } \\
\text { Intelligence }\end{array}$ & $\begin{array}{c}\text { Representative } \\
\text { Application }\end{array}$ \\
\hline \hline Blip-tronic 3000 [7] & Webcam & No & No & Yes & No & Robots & Music \\
\hline Diamond Touch [19] & Capacitance & Yes & Yes & No & N.A & No & Cartography \\
\hline Magets [56] & Fiber Optical / DSI & Yes & No & Yes & No & No & Proof of concept \\
\hline ReacTable [30] & Rear DI & Yes & No & Yes & No & No & Music \\
\hline TangiSense & RFID & No & Yes & Yes & Yes & Agents & Traffic Simulator \\
\hline
\end{tabular}

We have chosen an interactive table, called TangiSense. This table is not tactile, unlike the most of the interactive tables on the market and in the scientific literature. TangiSense table presents direct interaction via tangible objects. The TangiSense table has one advantage: this table detects overlapping objects, by using RFID technology. This technology can also store information about the different objects (e.g., size, history, behaviour). This solution allows the object to "agentify" by associating it with an agent during the simulation.

Figure 3 shows the TangiSense interactive table, which is made up of "tiles", each tile contains 64 antennas $(8 \times 8)$ and measures $2.5 \mathrm{~cm}$ square, while the table measures $1 \mathrm{~m} \times 1 \mathrm{~m}$. Designed by the RFIdees company ${ }^{3}$, this table is operational, but its hardware is constantly evolving. It is now a question of designing and developing the operating software for the table and the associated objects. With these objects, the users around the table are able to interact and work collaboratively with applications (e.g., simulation, design or production tasks, games), using physical objects.

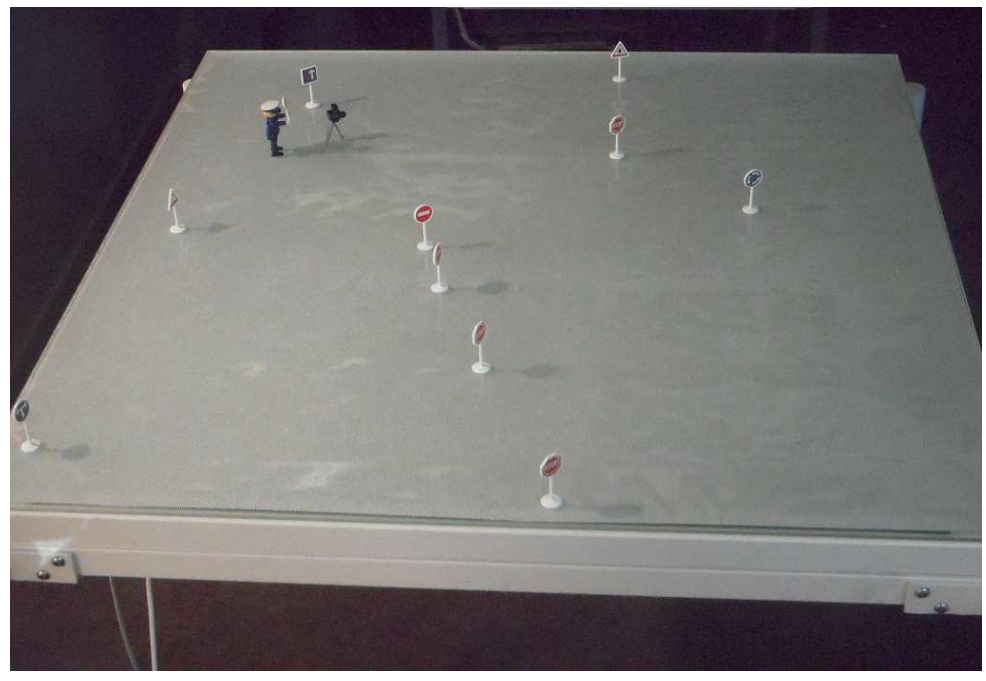

Figure 3: TangiSense interactive table equipped with RFID technology

As mentioned earlier, the objects used on our interactive table can be virtual or tangible, with the latter being physically accessible and easy to handle by the users. We call the visual objects video-projected on the table "virtual

\footnotetext{
${ }^{3}$ www.rfidees.fr
} 
objects". To accomplish the video projection, two technologies are possible on the TangiSense table. Either a set of LEDs is placed on the surface of the table or a video projector is used. The table is fitted with RFID antennas, which make it possible to detect the tangible objects that have RFID tags, which can store information, such as the history of the object's movements or the users' or objects' authentification information. During the initialization, the application associates the tags to one or several tangible objects.

The software architecture selected includes several layers (Figure 4):

- the hardware, which is the table itself.

- the middleware detects tangible objects, each equipped with one or more tags, handles events associated with the objects and communicates the modifications of the objects' positions to the multi-agent system.

- the Multi-Agent System (MAS) layer (presented in section 4.2) which brings reasoning capacities into the system. The MAS has a total view of the virtual and tangible objects that make up its environment. The organization [1] between the agents makes it possible for the objects to be intelligently managed and to assign roles to the objects.

- the Human-Computer Interaction (HCI) layer, which communicates with the users and which allows virtual information to be transmitted (e.g., the user's movement of a virtual object).

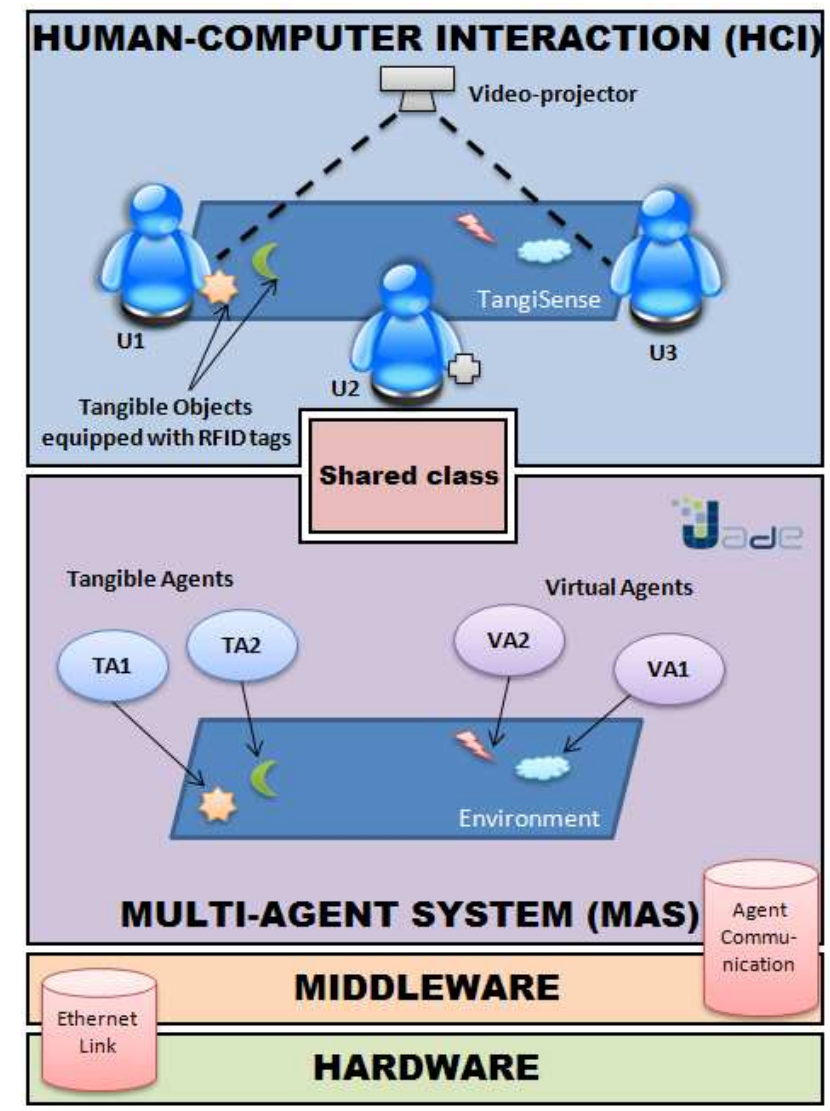

Figure 4: Software architecture of the TangiSense Table

Our research is focused on the $M A S$ and the $H C I$ layers. The $M A S$ manages the intelligent aspects of tangible and virtual objects, which can hold multiple roles dynamically. The $H C I$ uses the RFID technology as a basic capture mechanism for context-awareness [32]. In the next section, we present a multi-agent system designed especially for interactive tables. This system adds an intelligent layer for this new type of interaction platform. 


\section{Proposition of a multi-agent architecture for simulation on interactive table}

Since the rise of mobile platforms and new interaction surfaces (e.g., interactive table), we saw that it was advantageous to predict the adaptation to the $H C I$ context. The state of the art in section 2.1 proposed a set of criteria (Figure 2). Platforms like interactive tables were then considered. In this section, we consider the characteristics of interactive tables and complete the context model produced by the new criteria. These developments are outlined in bold-face in Figure 2. These criteria (see §4.1) are then integrated into our multi-agent architecture, which will be used to provide abilities to interact with our TangiSense interactive table.

\subsection{A model of context-awareness enriched to interactive tables}

Compared to the usual platforms (e.g., PC, laptop, PDA), one of the characteristics of interactive tables is that they can be multi-user. An attribute that allows the application to know whether or not the table is multi-user can be added to the Platform Class (Figure 2). Thus, several users can use the application at the same time. These users will have certain relationships that will modify the way that they collaborate and interact. A social link attribute is thus added to our model, which allows the application to know the types of relationships the users maintain.

Moreover, using interactive tables results in a new way of interacting and/or collaborating. This collaborative process needs new context criteria. In this paper, we do not focus on the platform changes; we only consider the context-awareness involved in using the table.

Generally, the context is taken into account by an application, which is adapted to the platform, the environment, and a user. Here, the applications are adapted to the environment, to the users but also to their position in relation to the table. We think that in such a context of use, the user's position is quite important, influencing the platform (i.e., the display) itself. In fact, the context will not be the same if the user is positioned on one side of the table or on the opposite side. An adaptation may be necessary to allow the user to work correctly according to his/her position on the table. These adaptations can be determined during a collaborative use of an interactive table. Each user must be able to work on his/her own space, which is allocated from the common space.

For the specific case of interactions with an interactive table, a criterion can be added to the model to know whether or not the environment is collaborative. This criterion is placed in the environment category rather than in the platform category because the platform makes collaboration possible, but the environment determines whether or not the collaboration is real. This collaborative environment can be distinguished by two characteristics that will influence the interactions:

- co-localized collaboration, which happens when the users use the table to collaborate;

- distant collaboration, which happens when the users have several distant platforms or the users use the table and another distant platform (e.g., another table or other platforms).

A contrario, the location is an unnecessary context criterion because the interactive table is generally not mobile, but it can be also added to the model.

According to our model (Figure 2), two kinds of objects can be used with interactive tables: virtual objects and tangible objects. In our case, the interactive table contains a set of RFID antennas, which allow the simultaneous use of virtual and tangible objects.

We describe the multi-agent architecture that brings the context-awareness mechanisms to the interactive table.

\subsection{Description of a multi-agent architecture}

The multi-agent system manages the behavior of the tangible objects moving on the table and of the virtual objects (i.e., informational deliberative entities) that are used to implement the table. As shown in Figure 4, we associate an agent to each tangible object [33], and to each interactive virtual object displayed on the table. Information coming from several RFID readers is transmitted by the middleware; this information is used by agents to build their views of their environment (as in Vrba et al. [52]). In order to design a MAS that controls the behavior of tangible and virtual objects for a given application, we need to define the relationships between the different types of agents and the functional roles that they have to play according to the application to be instantiated. 


\subsubsection{System organization}

To define the relationships between agents and their respective roles, we propose to use the class diagram given in Figure 5, which establishes links between agents and roles. This diagram is inspired by the research proposed by Odell et al. [38, 39]. The particularities and properties of the interactive table's applications led us to define a class of agents (MAM4ITAgent: Multi-Agent Model For Interactive Table) instantiated by all the agents used for these different applications.

We propose a SituatedAgent class that can be extended by either a TangibleAgent class for agents (associated to a real tangible object) or a VirtualAgent class for agents (associated to a virtual object projected on the interactive table). These agents are located in a Cartesian plane that represents the environment defined by the interactive table. A ConnectionAgent plays the scribe's role and contains information on the position (i.e., cartesian coordinate on the plane) and internal addresses of system agents (e.g., FIPA's Management Service Agent ${ }^{4}$ ).

A MAM4IT Agent possesses a list of role that can be initially empty or not. Indeed, the list of roles may dynamically evolve while the application is running:

- through self-adaptation (depending on its perception of the environment, an agent can ask the RoleManager agent to retrieve a role, or it can inform the RoleManager agent that it is leaving a role);

- through agent interaction (an agent can receive a role from another agent); or

- through a direct action of a user (a user is allowed to add and/or remove roles by interacting with the agent through tangible objects).

Initially, roles are stored and managed by an agent that has the RoleManager role; this agent plays a kind of directory services role. Like the Directory Facilitator (e.g., yellow pages) recommended by the standard FIPA, this type of agent makes it possible to discover which agents play which roles or what are the roles played by a given agent. It contains the list of couples <agent, roles $>$ and the list of couples $<$ objectID, role $>$ because some tangible objects can represent roles that users add to situated agents.

\subsubsection{Knowledge and Context}

To model our multi-agent systems and the roles, we used the formalisms defined by Adam et al. [1], which are based on the roles, and we took inspiration from the task decomposition of Hannoun et al. [27]. Each agent in the MAS is composed of knowledge, states, messages, personal rules, and roles. This knowledge includes: social knowledge $(K S)$ relative to knowledge about other agents; environmental knowledge $(K E)$ relative to the objects perceived in the environment and the environment map; personal knowledge $(K P)$ that contain the agent goals and properties.

Each agent also contains, by definition, a perception function that allows the agent to update its knowledge and to receive messages. According to the agent's knowledge and messages, it activates some of its personal rules and some of its roles.

An agent rule is composed of a set of elementary tasks; each task has a priority level that is used to evaluate the agent's preference for this rule.

The role characterizes the ability of an agent to perform some specific tasks [57]. In our case, a role is also composed of sets of: social knowledge $(K S)$ relative to knowledge about other roles; environmental knowledge $(K E)$ relative to the objects needed to play the role; personal knowledge $(K P)$ that contain the role's objective, the conditions in which the role can be activated (pre-requirement) and the consequences of modifying the agent's state. We propose to define a role $R$ with the equation 1 .

The role contains also the context knowledge $K C^{\text {role }}$ that defines different interaction criteria, such as user competence, user position and collaborative environment. Of course, this knowledge relative to the context could be stored in the set of $K E$ of environmental knowledge, but we propose, in this project, to extract the context knowledge $K C$ in order to facilitate the definition of the roles, from the designer point of view.

A role encompasses a set of rules. We define a rule (see equation 2) as a set of behaviors associated to the role. A rule is composed of a name, a priority level, and a set of elementary tasks. The rules are chosen according to the agent's knowledge, issued from its perception of the other, of the environment and according to the rules priority level.

\footnotetext{
${ }^{4}$ Foundation for Intelligent Agents (FIPA) defines standards for $M A S$ - www.fipa.org
} 
Figure 5: MAS class diagram

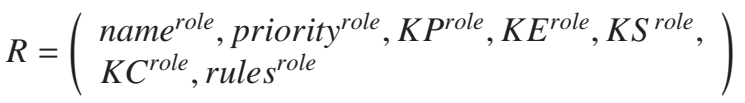

$$
\begin{aligned}
& \text { rule }=\left(\text { name }^{\text {rule }}, \text { priority }^{\text {rule }}, \text { tasks }^{\text {rule }}\right)
\end{aligned}
$$

For example, in road traffic management, the agents may have the role of driver (cf. Eq. 3).

$$
R=\left(\begin{array}{l}
\text { driver, priority }\{\}, K P\left\{\text { Driver }^{\prime} \text { s_license }\right\}, \\
\text { KE }\{\text { Roads; RoadS igns }\}, K S\{\text { Vehicles }\}, \\
\text { rules }\{\text { RespectStopS ign } ; \text { Bend } ; \text { Alert ... }\}
\end{array}\right)
$$

An agent receives the Driver role if he has the necessary prerequisites (e.g., a driver's license). Environmental knowledge of this role allow the agent to have a local representation of roads and road signs. We limit this knowledge because it is not necessary for the agent to know all the roads and traffic signs on the table. Therefore, the environmental knowledge is acquired by the agent through its field of vision. This local environment is sequentially updated at each movement. Social knowledge makes it possible to manage difficult situations with other agents (giving way to emergency vehicles, not colliding with the vehicle in front, etc.). The role is composed of a large set of rules that define a set of actions.

The notion of context of the $\mathrm{HCI}$ layer is a subset of the agent's environmental knowledge. The context knowledge $(K C)$ of an agent is composed of the triplet: <User, Platform, CollaborativeEnvironment $>$, as proposed in $\S 4.1$. 
We propose to use this triplet in the definition of $K C$ with $K C . U$ being the knowledge context of the User, $K C$.E the knowledge context of the nature of the Collaborative Environment, KC.P the knowledge context of the Platorm. Thus, we have: $K C=\{K C . U, K C . E, K C . P\}$.

The Context Knowledge allows an agent, through its roles, to adapt its interaction with the users and the other agents. Other interactions between agents are deduced from their classes. For example, an elementary principle between Virtual Agents and Tangible Agents relies on their interactions. A tangible agent/object can act on a virtual and/or tangible agent/object. In other words, a tangible agent/object's movement can push a virtual agent/object or another tangible agent/object, whereas this is not the case for a virtual agent/object. A virtual agent/object cannot act on a tangible agent/object.

The list of roles is dynamic for a given agent; this one can receive or reject some roles. We propose a role management procedure based on three major notions for a given role:

1. The pre-requirements that an agent must respect to be allowed to receive a role.

2. The implications that define a role's consequences on an agent (e.g., the addition, the modification of the agent's personal characteristics).

3. The coherence management of the role aggregation or combination process.

The pre-requirements and consequences are stored in the personal knowledge ( $\left.K P^{r o l e}\right)$ of the roles.

To manage the dynamic lists of agents' roles, we use the ManagerAgent in reference to the class diagram in Figure 5. The description of roles and agents are fundamental principles for developing an application. In the next section, we will use the proposed model in a case study highlighting these concepts.

\section{Case study: a simulation of road traffic management}

In this section, we present a case study related to the simulation of road traffic management. This contextawareness situation makes the various interactions with the interactive table visible (e.g., user collaboration or adjustment of the number of users and their positions around the table). In this simulation, we studied the possible interactions between virtual and tangible agents/objects. In this case, the $H C I$ and $M A S$ cannot be used independently and are integrated into the road traffic management simulation, which is described step by step.

\subsection{Consideration of the actors implied in the simulation}

In the research literature, the most common cases of context-awareness intervene during platform modifications (generally restricted) when users leave their personal computer and use a PDA or another mobile platform. In this paper, context-awareness does not focus on platform modifications. The interactions will focus on using the TangiSense table with tangible or virtual objects.

In this case study, a user, who is responsible for the infrastructure, initially occupies the interactive table's workspace alone. He can thus use all available space and all of the virtual and tangible objects needed in the design task. Two other users, a security expert and a site foreman, are able to work collaboratively with the person responsible for the infrastructure, but they have their own objects to use for the task (Figure 6). This change of context must be detected in order to adapt the interface to each expert user and to allow them to use their own objects.

The new users (i.e., the security expert and the site foreman) put their objects (equipped with one or more RFID tags) on the table. An object with a RFID tag can be identified and thus detected (We do not explain the process of authentification in this paper). The table detects the new users' objects and notifies the original user (i.e., the person responsible for the infrastructure) that he will not longer be working alone. The HCI then adapts the display of the preceding information so that the table is able to "share" the space among all of the users and associate each object to its $u^{2}{ }^{5}$. Since the users work collaboratively, it is not necessary to adapt the principal display ${ }^{6}$.

\footnotetext{
${ }^{5}$ In other types of application, it would be possible to highlight the objects of each expert by associating each tangible object with a virtual object (e.g., a different color halo under the objects of each expert).

${ }^{6}$ One can note however that the specific data to each expert must be marked according to the location of each user. For example, the security expert user must have safety markings for his/her position. Therefore, the context of use must be adapted [31].
} 


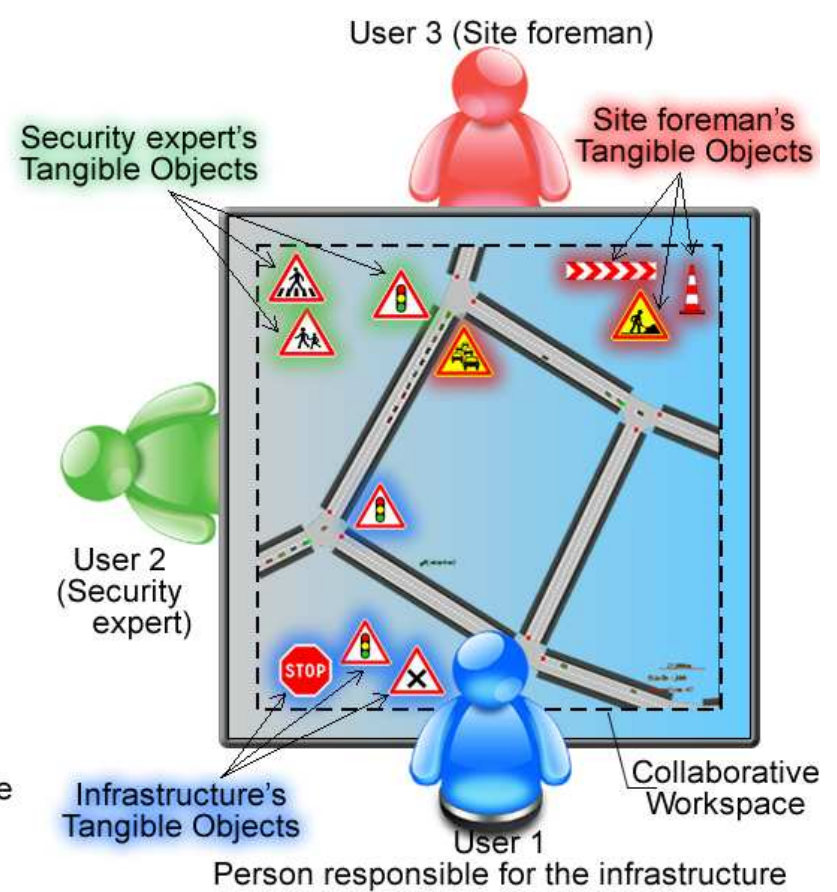

Figure 6: A context-awareness situation: individual (left part) and collaborative (right part) use of the TangiSense table with tangible objects

Figure 7 shows the context according to the classification system proposed in section 4.1. This figure shows the initial context (top table) and the modified context (bottom table). The number of users using the interactive table was modified. The profiles of the new users were then added, as well as the relationship between the users, if necessary. In this case, the user $(U 1)$ was assumed to be the colleague of $U 2$ and $U 3$. The environment became collaborative and co-localized.

This case study highlights the criteria proposed in section 4.1. It also justifies our context model and validates it through a simple example of interactive table use. Our example demonstrates that it is essential to adapt our model according to the use context of interactive tables, especially when using tangible objects.

\subsection{Road traffic management}

In the road traffic management simulation, vehicle agents represent drivers who drive on a road network. The road signs are the tangible agents/objects, and the vehicles and the road network are the virtual agents/objects (i.e., are shown on the table with a video projector). The rules for the driver role are defined according to the Highway Code. Vehicle agents have to choose the rules according to their interactions with the others, their perception of the other vehicle agents and the environment in which they are acting.

\subsubsection{Agent concepts useful for the simulation}

The simulation application proposes a set of facilities used by the person responsible for the infrastructure, the security and/or site foreman experts to manage road traffic. Its main objective is to optimize road traffic, like Balaji and Srinivasan [3] but using an interactive simulation, by avoiding traffic jams and by improving the emergency services.

For all applications based on the interactive table, we defined sets of tangible and virtual agents for the road traffic simulation:

- Tangible agents: they represent tangible objects equipped with one or more RFID tags. These objects correspond to road signs, traffic lights and some tangible actions. Some of the objects represent behaviors that can be associated to the agents. For instance, with a tangible object linked to an "ambulance" behavior, we can check the driver behavior and modify the behavior of a driver who has the "no breakdown" pre-requirement. We just have to place this object in the vehicle position of the driver projected on the table. 


\begin{tabular}{|c|c|c|}
\hline $\mathrm{U}$ & 1 & $\begin{array}{l}\text { User } 1 \\
\text { Vocation: person responsible for the infrastructure } \\
\text { Skill: consulting, network, economical conception, } \\
\text { etc. } \\
\text { UserPosition: in front of the table }\end{array}$ \\
\hline $\mathrm{E}$ & & $\begin{array}{l}\text { Time: 9:00 } \\
\text { Collaborative Environment: not applicable }\end{array}$ \\
\hline $\mathrm{P}$ & & $\begin{array}{l}\text { Multi-user: yes } \\
\text { Screen size: } 1 \mathrm{~m} \times 1 \mathrm{~m} \\
\text { Objects: tangible and virtual }\end{array}$ \\
\hline & & $\Downarrow$ Changes \\
\hline \multirow{3}{*}{$\mathrm{U}$} & 1 & $\begin{array}{l}\text { User } 1 \\
\text { Relation: } U 1 \text { colleague of } U 2 \text { and } U 3 \\
\text { UserPosition: in front of } U 3 \text {, right of } U 2\end{array}$ \\
\hline & 2 & $\begin{array}{l}\text { User } 2 \\
\text { Vocation: security expert } \\
\text { Skill: security, town-planning, organization } \\
\text { UserPosition: right of } U 3 \text { to the left of } U 1 \\
\text { Relation: } U 2 \text { colleague of } U 1 \text { and } U 3 \\
\end{array}$ \\
\hline & 3 & $\begin{array}{l}\text { User } 3 \\
\text { Vocation: site foreman } \\
\text { Skill: civil engineering } \\
\text { UserPosition: left of } U 2 \text { in the front of } U 1 \\
\text { Relation: } U 3 \text { colleague of } U 1 \text { and } U 2\end{array}$ \\
\hline $\mathrm{E}$ & & $\begin{array}{l}\text { Time: } 14: 00 \\
\text { Simulation speed: } * 2 \\
\text { Simulation speed: } * 5 \\
\text { Collaborative Environment: colocalised }\end{array}$ \\
\hline $\mathrm{P}$ & & $\begin{array}{l}\text { Objects: tangible and virtual (road signs, traffic } \\
\text { lights, roads, intersections, cars, etc.) }\end{array}$ \\
\hline
\end{tabular}

Figure 7: A context-aware situation: the state of the environment based the context model; Top: one user $(U 1)$; Bottom: three users $(U 1, U 2, U 3)$

- Virtual agents: projected on the table, they represent driver agents and service agents. For example, the Genius agent collects information about agent positions, informs agents about their environment, and transfers information to the HCI layer; and the roles Manager agent stores and/or transmits the different roles used while the application is running.

The context model (Figure 7) is included in the role description. It is updated when the middleware layer gives information (i.e., addition, withdrawal, displacement) to a tangible agent about a modification of the tangible object to which this agent is linked. This tangible agent can switch the user, the platform or the environment. In the road traffic simulation, the environmental context allows the speed at which the simulation progresses or the level detail of roads to be described, for example. These changes in situations are generally caused by the users and their skills, which allow them to test new situations. The number of users around the table and their skills can interact with the agent roles. Whenever a change is detected, information is sent to each agent to update their knowledge base. Depending on the situation, the drivers (vehicle agents) must adapt their behavior and if necessary compute new ways to reach their objectives.

Other tangible objects can also be used to create new vehicles on the table (i.e., the users put the stamp on the table) or to zoom in so to have an overview of traffic or of a specific part of the environment.

The environmental knowledge of vehicle agents is used to represent roads and road signs in the vicinity. The road network is stored in the environmental knowledge of vehicle agents as a weighted graph; it contains the directions, the speed limits, and the number of lanes. Social knowledge allows critical situations to be managed in terms of other agents (e.g., giving way to vehicles that have priority) [20].

For instance, RespectS topS ign rule relies on the following action plan consisting initially in stopping the vehicle 
and the driver looking left and right and then acting on traffic. The rules of a role are sorted by their level of priority. This priority is dynamic and computed at run time. For example, at a given time, a rule that we call bend reliant on the following plan consisting in calculating the bend degree and adjusting the car speed and finally turning the wheel can be subject to another rule (for example, RespectStopSign has priority over Bend) to avoid conflict.

\subsubsection{Illustration and validation on a scenario}

The scenario shows an example of a road traffic microscopic simulation (in the sense of [35]). The road map and the vehicles are projected on the interactive table by the HCI layer. The users can interact with this map by adding/removing/moving tangible objects equipped with RFID chip(s); these objects represent road signs, but some of them can be used to slow down the simulation, zoom in on/out from the map, or move the map.

When a vehicle agent is at an intersection, it receives messages from the tangible objects in the local environment that represents the road signs and adapts its behavior according to its state, its roles and its local environment. The user can move the road sign to different intersections to see the impact on road traffic.

The MAS manages messages from the interactive table, in addition to inter-agent messages. When the user moves an object on the surface of the table, the RFID antennas, composed of nine active zones, send the object positions to the MAS.

To illustrate the interactions with the tangible objects, the virtual objects and the users, we propose a scenario making it possible to follow the collaboration of three field experts, implied in the "road traffic management" (Table 2). This scenario illustrates an extract of a work session exploiting a road traffic simulation in the town of Valenciennes, in the north of France. The three experts must consider various possibilities concerning the installation of crossroads and maximum speeds for certain roads related to the crossroads.

Table 2: Detailed stages of the scenario

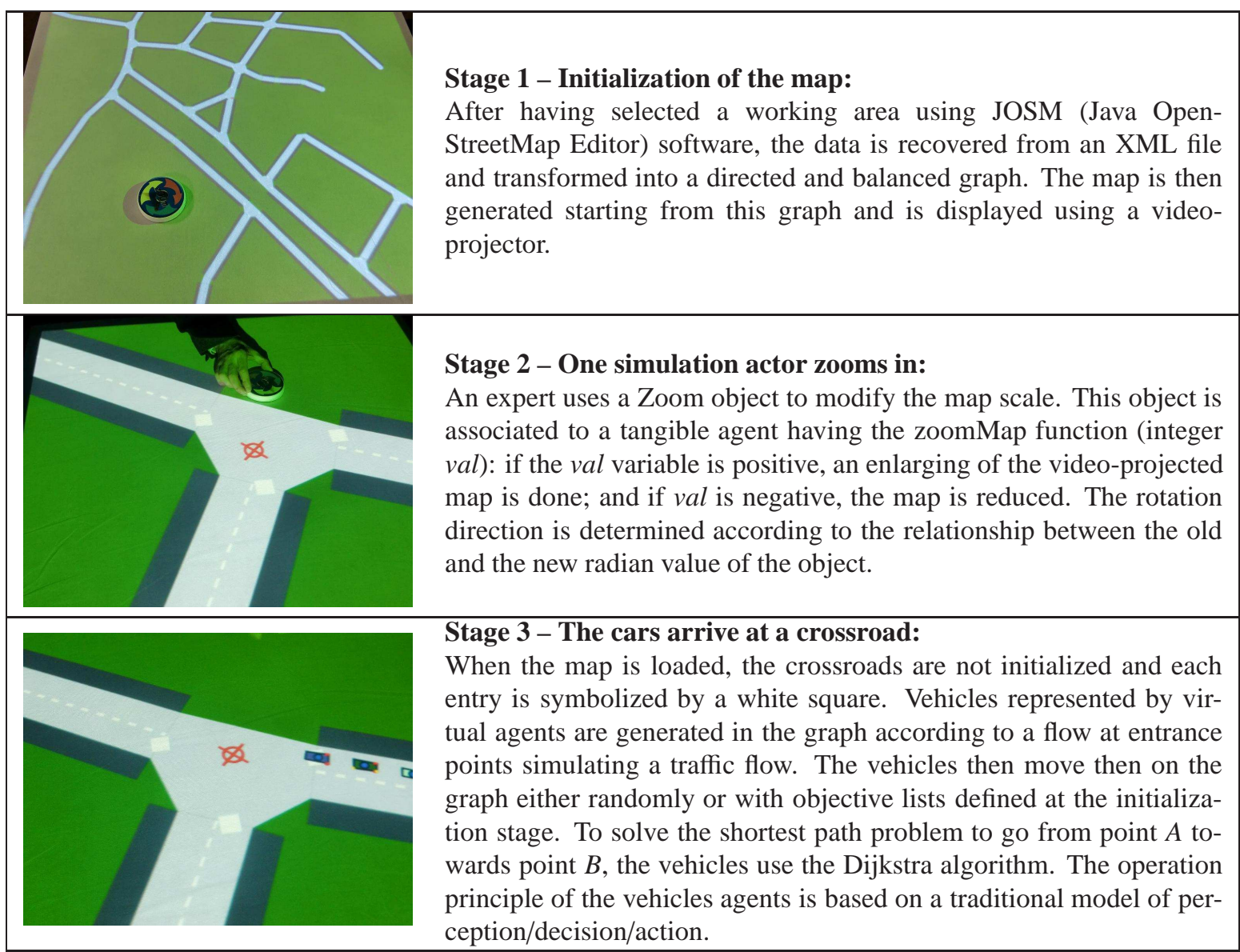


Stage 4 - The security expert places traffic lights objects at each crossroad:

A virtual agent is in charge of a crossroad and manages each entry. It prohibits or authorizes the vehicles to go through according to the road signs. On this figure, the security expert places traffic lights objects and initializes the various entries (an entry is initialized when a LEDs halo under the object becomes green). The agents in charge of traffic lights then communicate with the crossroad agent by sending a message to inform it of the signs which should be set up. In this situation the crossroad agent has as many behaviors as entries (here three). These behaviors are finite-state machines and make it possible to go from a green state to a red state, from a red state to an orange state, and from an orange state to a green state according to the evolution of time.

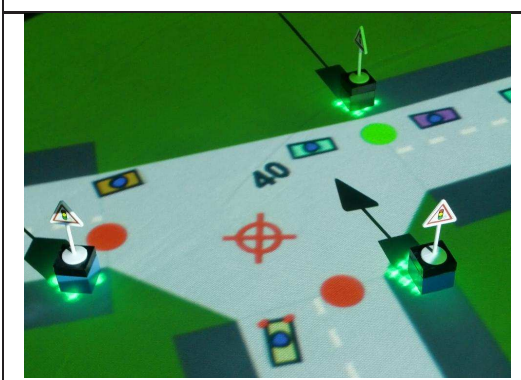

Stage 5 - Consideration taking of the traffic lights objects by the crossroads:

When the indication of the crossroads is set up, the traffic lights are activated one by one in a green state, which authorizes the vehicles to pass through. The setting up of this state implies the modification of the graph node associated to the crossroad entrance which the vehicles use to travel. The experts can analyze freely and easily the vehicle behaviors at this intersection (with the same types of concerns as in [13]).

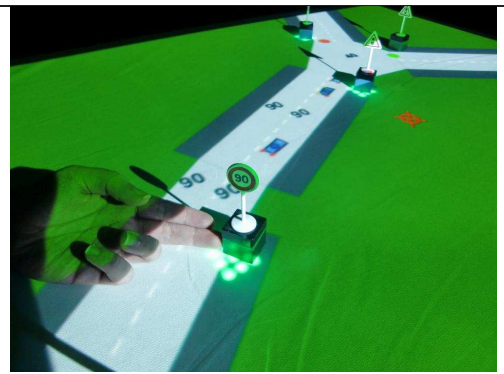

Stage 6 - The security expert proposes a speed limit of $90 \mathrm{~km} / \mathrm{h}$ before the crossroad:

The security expert places a road sign to limit the road to $90 \mathrm{~km} / \mathrm{h}$. The agent in charge of this object modifies the vertices of the global graph. The maximum speed that the vehicles can reach is updated from the road sign until the next intersection. The other vehicles update their speed as soon as they arrive near the limitation.

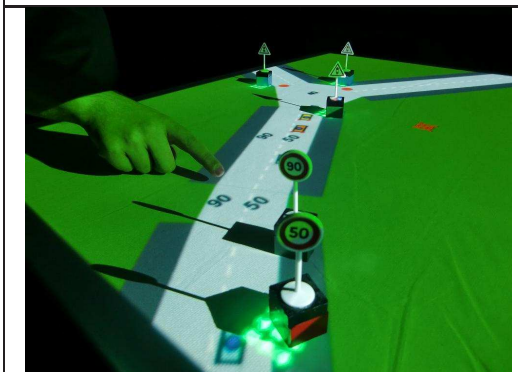

Stage 7 - The person responsible for the infrastructure proposes another speed limit of $50 \mathrm{~km} / \mathrm{h}$ when approaching a crossroad:

The person responsible for the infrastructure chooses to test the effect of the speed change of the road by limiting it to $50 \mathrm{~km} / \mathrm{h}$. When two tangible speed limit objects are placed on the same road, it is the last road sign placed which is taken into account by the system.

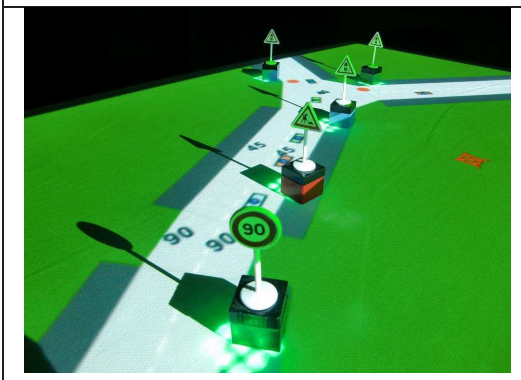

Stage 8 - The site foreman indicates that there will be some roadworks:

Finally, the person responsible for the infrastructure removes his panel because the site foreman has to indicate, using a warning road sign that there will be roadworks. This panel can be linked with the $90 \mathrm{~km} / \mathrm{h}$ speed limit and thus results in dividing the maximum speed by two (choice of the developers of the simulator). Consequently, the vehicles must slow down and go from $90 \mathrm{~km} / \mathrm{h}$ to $45 \mathrm{~km} / \mathrm{h}$ starting from new sign.

The proposed application is in the prototyping stage. It is written in Jade [6], a FIPA standard software imple- 
mented in Java, and used to simplify the deployment for the multi-agent applications. This tool allows without effort to create and activate the different agents, and to process exchanged messages between these agents.

\section{Discussion}

Although interactive tables are beginning to emerge from academic and industrial laboratories, and these tables are beginning to be commercialized, few (or none) software products are provided with the hardware. In addition, most software applications are musical applications, games, and applications managing multimedia data, especially pictures. With respect to industrial problems, the simulation is very little, or not at all, dealt with in interactive tables, while the economic stakes are potentially very important.

The simulation application is significant for the following reasons. It shows that the interactive table, coupled with tangible objects (with RFID technology, in our case) is a natural support to interactions between different users. This work seems to be coherent with other approaches $[29,9,36]$, which have contributed to tangible interactions, whatever the technology used. It also shows that the consistency of the information related to the simulation and the interactions in different contexts, which may vary considerably during a simulation session, and between tangible and virtual objects, is effectively supported by the multi-agent system. To our knowledge, this approach has never been used yet during the simulation of interactive table. Moreover, it can be reused for other applications, which are not described in this paper. We have implemented other applications that are different from traffic simulation, using a multi-agent approach for this interactive table [31].

Moreover, in most of the research work, the researchers do not explicitly take into account the decision-makers in the traffic simulation. Nevertheless it is important if one considers situations in which the decision-makers have to make choices among several alternatives (for example to study the environmental impact of different possible modifications in the road infrastructure). The underlying question could be expressed as the way to coordinate several human actors, each with specific functions, who must meet the requirements of their complex tasks. So we have proposed in this paper to represent different human actors (person responsible for the infrastructure, security expert, site foreman) acting with their tangible (i.e., physical) objects, each of these actors having their own goals to achieve (different context of use). The interactions between these different human actors depend not only on the direct actions on the agent-based simulation (in particular in the application presented in the paper, on the infrastructure, concerning the safety problems and considering the constraints linked to roadworks), because they are also the result of direct verbal communications. The modification of the infrastructure is thus directly taken into account by the simulated agents (vehicles, traffic lights, etc.); the response by a mathematical approach would not usually be immediate.

More generally, the use of interactive tables based on a MAS approach leads to analysis about people interacting with agents, which have not yet been explicitly considered in the literature (as regards neither interactive tables nor multi-agent approaches). Indeed we are confronted with problems, which besides being very difficult to analyze, involve mutual influence of decisions. The human decision-makers take decisions in a more or less collaborative way (that can be considered as a system of decision-makers); one can consider also that they propose implicitly or explicitly behavioral rules to be applied in the studied system (in our case the agent-based traffic system). At the level of the studied system, the agents react immediately to the environment; they can also generate other constraints, which have to be treated by the human decision-makers. It becomes clear that in this way, each system (MAS, group of decision-makers around the table) influences the others mutually. Such new simulation possibilities clearly show that a new research field is now open, regarding simulation on an interactive table, in collaborative contexts involving humans and software agents.

In short, we suggest a new research path for proposing and evaluating new simulators with interactive tables, in which the objects involved in the simulation can be both tangible and virtual objects. In this context, the users, through natural interactions, may themselves become the actors of the simulation.

\section{Conclusion}

In this paper, we proposed a new type of simulation. It relies on an interactive table, called TangiSense, and a set of tangible objects. These objects can be used and manipulated by a group of users, who are the decision makers, cooperating together around a given problem (e.g., for the design of a complex system). 
The RFID technology makes it possible to trace objects and to transmit information. We presented a state of the art about context and context-awareness (e.g., interaction context or context of use). We proposed a context model based on this state of the art and enhanced with new context criteria for using the TangiSense interactive table.

We proposed a multi-agent system appropriate for managing smart objects and the interaction context. This $M A S$ can be used with any application. It can combine different roles for the agent responsible for managing virtual or tangible objects. A case study in road traffic management simulation was proposed in order to illustrate the contextawareness mechanism and the multi-agent system.

This simulator allows different users to work together cooperatively at the interactive table by directly manipulating physical objects of a road infrastructure, which are part of tangible components of the simulation (e.g., traffic signs, lights). The objective is now to develop other demonstrators of new types of simulation systems, allowing interactions between several types of users. Many evaluations can also be envisaged.

\section{Acknowledgements}

This research was partially financed by the French Ministry of Education, Research \& Technology, the Nord/Pasde-Calais Region, the National Center for Scientific Research (CNRS), the FEDER program, the International Campus on Safety and Intermodality in Transportation (Plaiimob project), and the French National Research Agency (ANR TTT and IMAGIT projects, financial IMAGIT support: ANR-10-CORD-017). The authors would like to thank the partners with whom we collaborated on the TTT and IMAGIT projects: LIG, RFIdées, the CEA and Supertec. The authors thank also the anonymous reviewers for their numerous constructive remarks.

\section{References}

[1] Adam, E., Grislin Le-Strugeon, E., Mandiau, R., 2008. Flexible hierarchical organisation of role based agents. SASOW '08: Proc. of the Second IEEE International Conference on Self-Adaptive and Self-Organizing Systems Workshops, 186-191.

[2] AIMSUM2, 1999. V3.3 user's manual. Tech. rep., Getram Transport Simulation Systems.

[3] Balaji, P., Srinivasan, D., 2011. Type-2 fuzzy logic based urban traffic management. Engineering Applications of Artificial Intelligence 24 (1), 12-22.

[4] Banks J. Handbook of simulation: principles, methodology, advances, applications and practice. Wiley-Interscience, 1998.

[5] Bazzan, A., 2005. A distributed approach for coordination of traffic signal agents. Journal of Autonomous Agents and Multi-Agent Systems 10(2), 131-164.

[6] Bellifemine, F., Poggi, A., Rimassa, G., 2001. Developing multi-agent systems with a FIPA-compliant agent framework. Software Practice and Experience 31 (2), 103-128.

[7] Benett, P., Toru, S., Tutte-Scali, L., 2005. Blip-Tronic 3000. http://www.petecube.com/bliptronic3000/

[8] Bhouri, N., Balbo, F., Pinson, S., 2012. An Agent-Based Computational Approach for Urban Traffic Regulation. Progress in Artificial Intelligence 1(2), 139-147.

[9] Blackwell, A., Fitzmaurice, G., Ishii, H., 2007. Tangible user interfaces in context and theory. Proceedings CHI 2007, California, USA, May, ACM Press.

[10] Browne, D., Norman, M., Riches, D., 1990. Why Build Adaptive Interfaces. In: Browne, D., Totterdell, P., Norman, M. (Eds.), Adaptive User Interfaces. Academic Press, pp. 15-57.

[11] Calvary, G., Coutaz, J., Thevenin, D., 2001. Supporting Context Changes for Plastic User Interfaces: a Process and a Mechanism. In: Proc. HCI-IHM 2001, A. Blandford, J. Vanderdonckt, P. Gray Eds.,BCS conference series, Springer Publ. pp. 349-363.

[12] Calvary, G., Demeure, A., Coutaz, J., Dâassi, O., 2004. Adaptation des Interfaces Homme-Machine à leur contexte d'usage. Revue d'intelligence artificielle 18 (4), 577-606.

[13] D’Ambrogio, A., Iazeolla, G., Pasini, L., Pieroni, A., 2009. Simulation model building of traffic intersections. Simulation Modelling Practice and Theory 17 (4), 625-640.

[14] de Cote, E. M., Lazaric, A., Restelli, M., 2006. Learning to cooperate in multi-agent social dilemmas. In: Proceedings of the fifth international joint conference on Autonomous agents and multi-agent systems. AAMAS '06. ACM, New York, NY, USA, pp. 783-785.

[15] Davidsson, P., Henesey, H., Ramstedt, L., Tornquist, J. and Wernstedt, F., 2005. Agent-based approaches to transport logistics. In: Application of Agent Technology Traffic and Transportation (ATT 2005), 1-16.

[16] Dey, A. K., Mankoff, J., 2005. Designing mediation for context-aware applications. ACM Trans. Comput.-Hum. Interact. 12 (1), 53-80.

[17] Dey, A. K., Salber, D., Futakawa, M., Abowd, G. D., 1999. An Architecture To Support Context-Aware Applications. GVU Technical Reports.

[18] Dey, A. K., Sohn, T., Streng, S., Kodama, J., 2006. iCAP: Interactive Prototyping of Context-Aware Applications. In: Proc. of the fourth international conference on pervasive computing. Dublin, Ireland, pp. 254-271.

[19] Dietz, P., Leigh, D., 2001. DiamondTouch: a multi-user touch technology. In: Proc. of the 14th annual ACM symposium on User Interface Software and Technology. New York, NY, USA, pp. 219-226.

[20] Doniec, A., Mandiau, R., Piechowiak, S., Espié, S., 2008. A behavioral multi-agent model for road traffic simulation. Engineering Applications of Artificial Intelligence 21 (8), 1443-1454. 
[21] Dressner, K., Stone, P., July 2004. Multi-agent traffic management: A reservation based intersection control mechanism. In: in Proc. of the 3rd International Conf. On Autonomous Agents and Multi-agent Systems: AAMAS 04, New-York (USA). pp. 530- 537.

[22] Dressner, K. and Stone, P., 2008. A multi-agent approach to autonomous intersection management. Journal of Artificial Intelligence Research, 591-656.

[23] Ehlert, P. A. and Rothkrantz, L. J., 2001. Microscopic traffic simulation with reactive driving agents. In IEEE Intelligent Transportation Systems Conference Proceedings, 861-866.

[24] Ferber, J., 1999. Multi-Agent Systems: An Introduction to Distributed Artificial Intelligence, Addison Wesley.

[25] Ferreira, E. D., Subrahmanian, E., Manstetten, D., 2001. Intelligent agents in decentralized traffic control. In IEEE Intelligent Transportation Systems Conference Proceedings. Oakland (CA), USA, pp 707-711,

[26] Gárate, A., Herrasti, N., López, A., 2005. Genio: an ambient intelligence application in home automation and entertainment environment. In: Proceedings of the 2005 joint conference on Smart objects and ambient intelligence: innovative context-aware services: usages and technologies. sOc-EUSAI '05. ACM, New York, NY, USA, pp. 241-245.

[27] Hannoun, M., Sichman, J. S., Boissier, O., Sayettat, C., 1998. Dependence relations between roles in a multi-agent system: Towards the detection of inconsistencies in organization. In: Berlin, S. (Ed.), Multi-Agent Systems and Agent-Based Simulation. Vol. 1534. pp. 169-182.

[28] Hartmann A.K. A practical guide to computer simulation. World Scientific Publishing Company, 2009.

[29] Ishii, H., Ullmer, B. (1997). Tangible Bits: Towards Seamless Interfaces Between People, Bits, and Atoms. Proceedings CHI 1997, $234-41$.

[30] Jordà, S., Kaltenbrunner, M., Geiger, G., Alonso, M., 2006. The reacTable: a tangible tabletop musical instrument and collaborative workbench. In: SIGGRAPH '06: ACM SIGGRAPH 2006 Sketches. New York, USA, pp. 91.

[31] Kubicki, S., Lepreux, S., Kolski, C., 2011. RFID-driven situation awareness on TangiSense, a table interacting with tangible objects. Personal and Ubiquitous Computing DOI: 10.1007/s00779-011-0442-9.

[32] Kubicki, S., Lepreux, S., Kolski, C., Caelen, J., 2010. Towards New Human-Machine Systems in contexts involving interactive table and tangible objects. In: 11th IFAC/IFIP/IFORS/IEA Symposium on Analysis, Design, and Evaluation of Human-Machine Systems. Valenciennes, France.

[33] Lebrun, Y., Adam, E., Kubicki, S., Mandiau, R., April 2010. A Multi-agent System Approach for Interactive Table Using RFID. In: 8th International Conf. on Practical Applications of Agents and Multi-Agent Systems (PAAMS 2010). Springer.

[34] Lieberman, E., Rathi, A., 1997. Traffic flow theory. Oak Ridge National Laboratory, Chapter Traffic simulation, 1997.

[35] López-Neri, E., Ramírez-Treviño, A., López-Mellado, E., 2010. A modeling framework for urban traffic systems microscopic simulation. Simulation Modelling Practice and Theory 18 (8), $1145-1161$.

[36] Manches, A., Malley, C. O., Benford, S. (2009). Physical Manipulation: Evaluating the Potential for Tangible Designs, 77-84. Proceedings TEI Conference 2009, Cambridge, UK, feb.

[37] Meir, R. and Rosenschein, J-S, 2010. A game theoretic approach to leasing agreements can reduce congestion.. In: 6th Workshop on Agents in Traffic and Transportation, co-located with the 8th International Joint Conference on Autonomous Agents and Multi-Agent Systems (ATT@AAMAS 2010), Toronto, Canada, pp. 21-27.

[38] Odell, J. J., Parunak, H. V. D., Fleischer, M., juillet 2003. Modeling agent organizations using roles. In: Software and Systems Modeling. Vol. 2. pp. 76-81, computer Science.

[39] Odell, J. J., Parunak, H. V. D., Fleischer, M., 2003. The role of roles in designing effective agent organizations. In: Software Engineering for Large-Scale Multi-Agent Systems, LNCS 2603. Vol. 2603. pp. 27-38.

[40] Paruchuri, P., Pullalarevu, A. R., Karlapalem, K., 2002. Multi agent simulation of unorganized traffic. Proceeding of The Internationnal Joint Conference on Autonomous Agents and Multi-agent Systems (AAMAS’02). Bologne, Italie, pp. 176-183.

[41] Pascoe, J., 1998. Adding Generic Contextual Capabilities to Wearable Computers. In: ISWC '98: Proceedings of the 2nd IEEE International Symposium on Wearable Computers. IEEE Computer Society, Washington, DC, USA, pp. 92-99.

[42] Pascoe, J., Thomson, K., Rodrigues, H., 2007. Context-Awareness in the Wild: An Investigation into the Existing Uses of Context in Everyday Life. In: OTM Workshops. pp. 193-202.

[43] Reece, D. A., Shafer, S. A., 1993. A computational model of driving for autonomous vehicles. Transportation Research 27 (1), $23-50$.

[44] Rey, G., Coutaz, J., 2004. The Contextor Infrastructure for Context-Aware Computing. In: Workshop on Component-Oriented Approaches to Context-Aware Computing(COACAC, ECOOP). Vol. LNCS 3344. Springer Verlag.

[45] Ruskin, H. J., Wang, R., 2002. Modelling traffic flow at an urban unsignalized intersection. In: Proceedings of International Conference on Computational Science, pp. 381-390.

[46] Schilit, B., Adams, N., Want, R., 1994. Context-Aware Computing Applications. In: WMCSA'94: Workshop on Mobile Computing Systems and Applications. IEEE Press, Santa Cruz, CA, pp. 85-90.

[47] Simonin, J., Carbonell, N., 2007. Interfaces adaptatives - Adaptation dynamique à l'utilisateur courant. In: Saleh, I., Regottaz, D. (Eds.), Interfaces numériques. Hermès, Paris, pp. 37-54.

[48] Thevenin, D., Coutaz, J., 1999. Plasticity of User Interfaces: Framework and Research Agenda. In: Johnson, C. (Ed.), Interact'99. IFIP IOS Press, Edinburgh, pp. 110-117.

[49] Trannois, H., Lebrun, A. and Deleage, J., 1998. A multi-agent framework for car traffic simulation. In: The Third International Conference and Exhibition on the Practical Application of Intelligent Agents and Multi-agent (PAAM).

[50] Vasirani, M. and Ossowski, S., 2011. A computational market for distributed control of urban road traffic systems. IEEE Transactions on Intelligent Transportation Systems 12(2), 313-321.

[51] Vissim, 2005. Vissim 4.10. User Manual. Technical Report. PTV Planung Transport Verkehr AG: Karlsruhe, Germany.

[52] Vrba, P., Macurek, F., Marík, V., 2008. Using radio frequency identification in agent-based control systems for industrial applications. Engineering Applications of Artificial Intelligence 21 (3), 331-342.

[53] Ward, A., Jones, A., Hopper, A., October 1997. A New Location Technique for the Active Office. IEEE Personnal Communications 4 (5), $42-47$.

[54] Weiser, M., 1999. The Computer for the 21st Century. SIGMOBILE Mob. Comput. Commun. Rev. 3 (3), 3-11.

[55] Weiss, G., 2000. Multiagent Systems: A Modern Approach to Distributed Artificial Intelligence, MIT Press. 
[56] Weiss, M., Schwarz, F., Jakubowski, S., Borchers, J., 2010. Madgets: actuating widgets on interactive tabletops. UIST '10: 23nd annual ACM symposium on User interface software and technology. New York, USA, pp. 293-302.

[57] Wooldridge, M., Jennings, N. R., Kinny, D., September 2000. The gaia methodology for agent-oriented analysis and design. Journal of Autonomous Agents and Multi-Agent Systems 3 (3), 285-312.

[58] Zhang, F., Li, J., and Zhao, Q. 2005. Single-lane traffic simulation with multi-agent system. In IEEE Conference on Intelligent Transportation Systems, 1183-1187.

[59] Zeddini, B., Zargayouna, M. and Yassine, A. 2010. Space and space-time organization model for the dynamic vrptw. In: 6th Workshop on Agents in Traffic and Transportation, co-located with the 8th International Joint Conference on Autonomous Agents and Multi-Agent Systems (AAMAS 2010), Toronto, Canada, 21-27.

[60] Ziegler B.P., Praehofer H., Kim T.G, 2000. Theory of modeling and simulation. Second edition, Academic Press, 2000. 